\title{
Publicidades activa y pasiva en contratación pública. Una panorámica autonómica española
}

\section{Active and passive advertising in public contracts. A Spanish regional overview}

\author{
Pilar Beltrán-Orenes; Esther Martínez-Pastor
}

Cómo citar este artículo:

Beltrán-Orenes, Pilar; Martínez-Pastor, Esther (2019). “Publicidades activa y pasiva en contratación pública. Una panorámica autonómica española”. El profesional de la información, v. 28, n. 3, e280319.

https://doi.org/10.3145/epi.2019.may.19

Artículo recibido el 18-12-2018

Aceptación definitiva: 16-04-2019

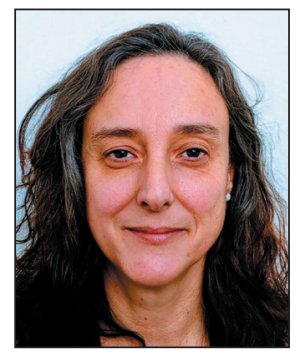

Pilar Beltrán-Orenes $\square$

https://orcid.org/0000-0002-1408-6365

Universidad Rey Juan Carlos

Campus de Fuenlabrada

Camino del Molino, $\mathrm{s} / \mathrm{n}$.

28943 Fuenlabrada (Madrid), España

pilar.beltran@urjc.es

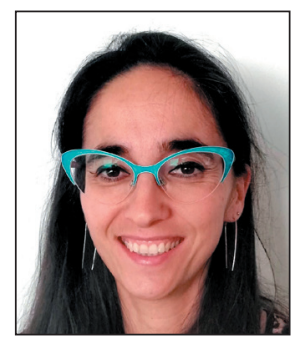

Esther Martínez-Pastor

https://orcid.org/0000-0002-2861-750X

Universidad Rey Juan Carlos

Campus de Fuenlabrada

Camino del Molino, s/n.

28943, Fuenlabrada (Madrid), España

esther.martinez.pastor@urjc.es

\section{Resumen}

La cronología de las principales normas en las que se recogen las publicidades activa y pasiva sobre contratación pública en España, tiene una historia anterior a la Ley 19/2013, de 9 de diciembre, de transparencia, acceso a la información pública y buen gobierno. La información contractual pública sujeta a obligaciones de transparencia por medios electrónicos empezó su andadura mucho antes que el resto de las informaciones públicas sujetas a transparencia. Este hecho ha tenido como resultado una notable diversidad en el progreso autonómico en la creación de plataformas para la publicidad activa contractual, y de los organismos y los órganos para el control de las publicidades activa y pasiva contractuales, respectivamente. El mapa autonómico actual es diverso, haciendo difícil el ejercicio de su control en transparencia, por lo que se propone una posible solución mediante el concepto de soft law de la Unesco.

\section{Palabras clave}

Transparencia; Publicidad activa; Publicidad pasiva; Autonomías; España; Consejo de transparencia y buen gobierno; Tribunal administrativo central de recursos contractuales; Tacrc; Plataforma de contratación del sector público; Soft law; Unesco.

\begin{abstract}
The chronology of the main rules in which active and passive advertising on public procurement in Spain are collected, are prior to Law 19/2013, of 9 December, of transparency, access to public information and good governance. Public contractual information by electronic means began much earlier than the rest of the public information subject to transparency. This has resulted in a notable diversity in regional progress in the creation of platforms for contractual active advertising, and of agencies and bodies for the control of active and passive advertising contracts, respectively. The current regional map is diverse being difficult to exercise its control in transparency. The article proposes a possible solution via the concept of Unesco's soft law.
\end{abstract}




\section{Keywords}

Transparency; Active advertising, Passive advertising; Regions; Spain; Transparency and Good Governance Council; Central Administrative Tribunal for Contractual Resources; Platform of public contracting; Soft law; Unesco.

\section{Introducción}

Existen varios trabajos centrados en el análisis del cumplimiento en transparencia a nivel autonómico en sus respectivos portales. Cabe destacar los realizados por:

- Curto-Rodríguez (2017): sobre la información contable-presupuestaria, transparencia de los cargos gubernamentales y suministro de información relativa al gasto público de los portales de datos abiertos, que elabora un ranking de las comunidades autónomas tras el análisis de veinte items;

- García-García y Curto-Rodríguez (2018): amplía la revisión a los portales de transparencia y web institucionales autonómicos.

- Sierra-Rodríguez (2017): en el que se analizan los organismos de garantía de la transparencia, tanto desde el Estado como de las autonomías, realizando un acercamiento sobre el campo de tutela en el que operan, su estructura y caracterización. Entre sus conclusiones destaca que la organización de los organismos de garantía de la transparencia en el ámbito nacional y autonómico es poco satisfactoria, además de complicar el panorama de la transparencia por sus diferentes organismos de control.

Se presenta una recopilación de los medios usados por los territorios autonómicos para la publicidad activa en materia de contratación, y los organismos y los órganos creados para el control de sus publicidades activa y pasiva. El objetivo es presentar el mapa de cumplimiento y de centralización, o descentralización autonómicos, siguiendo el hilo cronológico que las principales normas estatales han fijado sobre las publicidades activa y pasiva ${ }^{1}$, y su control en materia de contratación pública.

\section{Evolución de la publicidad contractual: hitos normativos}

Numerosos estudios académicos dejan patente la relevancia de la transparencia en la contratación pública.

Ésta tiene como antecedentes la Ley 30/1992, de 26 de noviembre de régimen jurídico de las administraciones públicas y del procedimiento administrativo común y la Ley 24/2002, de 27 de diciembre, de medidas fiscales, administrativas y de orden social, que preveían la incorporación de medios técnicos y la creación de registros telemáticos para la entrada o salida de cualquier escrito o solicitud (González-Alonso, 2009).

La Ley 11/2007, de 22 de junio, de acceso electrónico de los ciudadanos a los servicios públicos fortalece el principio de transparencia en contratación administrativa y permite un seguimiento de los contratos públicos (González-Alonso, 2009).

La Ley 30/2007, de 30 de octubre, de contratos del sector público (LCSP) (España, 2007) expresa la obligatoriedad de que las administraciones hagan públicas sus contrataciones (artículo 2), así como la creación de una plataforma de contratación pública del Estado para este fin en su artículo 309.1. Esta plataforma se pone a disposición de todos los organismos de la Administración General del Estado, para que incluyan la información relativa a sus licitaciones como anuncios previos, pliegos de contratación, adjudicaciones y su perfil de contratante (Fuentes-Bargues; González-Gaya; González-Cruz, 2015). En esta plataforma, las entidades u organismos autónomos y locales pueden publicitar sus contrataciones (Beltrán-Orenes; Martínez-Pastor, 2016). El perfil de contratante se convierte en la principal vía de publicidad contractual:

"La forma de acceso al perfil de contratante deberá especificarse en las páginas web institucionales que mantengan los entes del sector público, en la Plataforma de Contratación del Estado y en los pliegos y anuncios de licitación" (artículo 42).

El objetivo es asegurar la transparencia y el acceso a la información de la actividad contractual de las Administraciones Públicas (Canales-Gil; Huertas-Baraja, 2010).

Tres años después, la Ley 34/2010, de 5 de agosto, de modificación de las Leyes 30/2007, de 30 de octubre, de contratos del sector público, 31/2007, de 30 de octubre, sobre procedimientos de contratación en los sectores del agua, la energía, los transportes y los servicios postales y 29/1998, de 13 de julio, reguladora de la jurisdicción contencioso-administrativa para adaptación a la normativa comunitaria de las dos primeras (España, 2010) incorpora, entre los actos recurribles por la vía contencioso-administrativa, "los anuncios de licitación, los pliegos y los documentos contractuales que establezcan las condiciones que deban regir la contratación" (artículo 310.2a); así como la creación del Tribunal Administrativo Central de Recursos Contractuales (Tacrc), (artículo 311). De tal manera que la 
"jurisdicción contencioso-administrativa y el derecho administrativo vienen configurando el sistema de control de las adjudicaciones contractuales" (González-Varas-Ibáñez, 2010, p. 63).

Se da un paso más con la entrada en vigencia del Real decreto legislativo 3/2011, de 14 de noviembre, por el que se aprueba el texto refundido de la ley de contratos del sector público (España, 2011), que establece la exclusividad de la Plataforma de Contratación del Sector Público (PCSP) como medio de difusión de la contratación pública estatal. También establece la obligación de enlazar desde las sedes electrónicas de las entidades estatales su correspondiente perfil de contratante en dicha plataforma (artículo 334.1), con la finalidad de acreditar fehacientemente el inicio de la difusión pública de la información que se incluya en la misma (artículo 334.2). De este modo, los perfiles de contratantes del sector público estatal deberán integrarse en esta plataforma, gestionándose y difundiéndose exclusivamente a través de la misma (artículo 334.1). En las sedes electrónicas de estos órganos se incluirá un enlace a su perfil del contratante situado en la PCSP. Asimismo, la PCSP se interconectará con los servicios similares que hayan articulado las comunidades autónomas y entidades locales de acuerdo con los acuerdos que se realizan a tal efecto (artículo 334.5).

La Ley 19/2013, de 9 de diciembre, de transparencia, acceso a la información pública y buen gobierno (LTBG) (España, 2013) amplía el tipo de información que las administraciones públicas deben publicar en internet. Y establece la diferencia entre publicidad activa, la que deben publicar las administraciones públicas, y derecho de acceso a la información pública (publicidad pasiva, previa solicitud) (Guichot, 2014). En materia contractual se hace con el propósito de que los ciudadanos puedan tener conocimiento de las contrataciones públicas y exigir obligaciones (Carrascosa-Puertas, 2017; Blanes-Climent, 2014).

Para la consecución de la publicidad activa se crea el Portal de la Transparencia (PT) (artículo 10), en el que se incluirá toda la información exigida a los organismos y entidades de la Administración Pública (artículos 6-8), incluyendo la contractual (artículo 8.1 a). Se deja, no obstante, que la Administración General del Estado, las Administraciones de las Comunidades Autónomas y de las Ciudades de Ceuta y Melilla y las entidades que integran la Administración Local puedan desarrollar medidas complementarias, creando webs para la accesibilidad a esta información (artículo 10.3). Asimismo se establece el mecanismo de control de la publicidad activa, el Consejo de Transparencia y Buen Gobierno (CTBG), artículo 9.1, que velará por el cumplimiento de la Administración General del Estado de las obligaciones de publicidad activa recogidas en el Capítulo II de la LTBG. La LTBG no hace referencia al control de la publicidad activa de las administraciones autonómicas, sólo nueve de los organismos autonómicos creados ejercen la tutela sobre la misma (Sierra-Rodríguez, 2017).

En cuanto a la publicidad pasiva, derecho de acceso a la información pública, se perfilan los límites (artículos 12-16), el modo de ejercer el derecho (artículos 17-22) y el organismo ante el que reclamar, el CTBG (artículo 24). Y se expresa que

"las Administraciones Públicas incluidas en el ámbito de aplicación de este título establecerán sistemas para integrar la gestión de solicitudes de información de los ciudadanos en el funcionamiento de su organización interna” (artículo 21.1).

Los territorios autonómicos podrán firmar convenio con el CTBG, asumiendo el coste de esta asunción, o crear sus organismos propios (Disposición adicional cuarta).

Según la $L T B G$, en materia de contratación autonómica el organismo de control de la publicidad activa es el CTBG, o sus equivalentes autonómicos, previa firma de convenio; también se encargan de la pasiva, atendiendo a las reclamaciones que se presenten, junto con el Tacrc o sus homólogos autonómicos (España, 2010).

El solapamiento de las atribuciones del CTBG y el Tacrc (o de sus homólogos autonómicos) en materia de reclamaciones de publicidad pasiva contractual ha sido despejado, en principio, por la Ley 9/2017, de 8 de noviembre, de contratos del sector público, por la que se transponen al ordenamiento jurídico español las Directivas del Parlamento Europeo y del Consejo 2014/23/UE y 2014/24/UE, de 26 de febrero de 2014 (España, 2017). En ella se limita el papel del CTBG a la elaboración de informes que eximan de publicar algunos datos:

"los órganos de contratación deberán solicitar la emisión de informe por el [CTBG] a que se refiere la [LTBG], en el que se aprecie si el derecho de acceso a la información pública prevalece o no frente a los bienes que se pretenden salvaguardar con su no publicación, que será evacuado en un plazo máximo de diez días" (artículo 154.7).

Siendo esta labor exclusiva del CTBG, sin posibilidad de que la ejerzan sus homólogos autonómicos.

También se recoge en esta ley, como causa de nulidad de derecho administrativo, "la falta de publicación del anuncio de licitación en el perfil de contratante" (artículo 39.c), y se refuerza el perfil de contratante al que

"le otorga un papel principal como instrumento de publicidad de los distintos actos y fases de la tramitación de los contratos de cada entidad, así como la regulación del Registro de Contratos del Sector Público" (Preámbulo, IV).

Tal como señala Mellado-Ruiz (2018), la relevancia de la publicidad en esta Ley va más allá de la publicidad instrumental, al ser un mandato o directriz de optimización que todos los órganos de contratación pública deben cumplir. Por otra parte, la correcta aplicación de la Ley 9/2017, de 8 de noviembre, de contratos del sector público (España, 2017), hace necesaria la asunción de los principios imperativos de integridad y estrategia que ésta marca en materia contractual a 
las administraciones públicas (Martínez-Fernández, 2017; 2018). Y se enmarca, además, dentro de la gobernanza inteligente, a la que está dando lugar el uso de los datos masivos y abiertos.

“El avance de la gobernanza inteligente está estrechamente vinculado a la disponibilidad de datos de calidad que puedan ser fácilmente reutilizables y de capacidades adecuadas tanto en las administraciones públicas como en la ciudadanía, que permitan su análisis" (Cerrillo-Martínez, 2018).

Asimismo, se amplía la opción de crear nuevos tribunales administrativos en ayuntamientos considerados de gran población y en diputaciones provinciales (Pérez-Delgado; Rodríguez-Pérez, 2017). Siendo los distintos tribunales administrativos, central o autonómicos, o los futuros consistoriales o provinciales, los encargados de los recursos (publicidad pasiva) administrativos contractuales (artículo 46).

\section{Metodología}

Siguiendo la cronología de las normas ${ }^{2}$ y la importancia creciente de las publicidades activa y pasiva en ellas, los territorios autonómicos españoles deben cumplir los siguientes requisitos de publicidad en materia de contratación pública:

1. Dar acceso a su perfil de contratante desde la PCSP y publicar sus licitaciones en ella o en las autonómicas (España, 2007; 2011).

2. Dar acceso a su perfil de contratante desde sus sitios webs oficiales (España, 2007; 2011).

3. Tener un órgano para el control del derecho de acceso a la información contractual, publicidad pasiva, el Tacrc o el autonómico (España, 2010; 2017).

4. Tener un organismo autónomo que controle la publicidad activa, el CTBG o el autonómico (España, 2013).

5. Dar acceso al perfil de contratante desde el PT o los portales de transparencia autonómicos (España, 2013).

Los 5 items se han dividido en dos grupos temáticos. El primero relativo en exclusiva al perfil de contratante como instrumento esencial de la publicidad activa. Se ha buscado su acceso:

- desde el sitio web oficial autonómico (requisito 2);

- desde el portal de transparencia autonómico (requisito 5).

El otro grupo referido al progreso autonómico, presencia y tipo de:

- plataformas de publicidad activa (requisito 1);

- organismos de control de la publicidad activa (requisito 4) y órganos de control de la pasiva (requisito 3).

Se han recopilado los datos de cada ítem en todos los territorios autonómicos. Para ello se han revisado los 19 sitios web oficiales y portales de transparencia autonómicos, así como los sitios web de la PCSP, el CTBG y el Tacrc. Los resultados se han plasmado en un mapa por cada ítem y grupo de items, los dos grupos temáticos. Cuando el resultado de un ítem, o grupo, ofrecía un mapa igual a otro ya realizado, se ha optado por remitir a éste.
Los territorios autonómicos pueden publicar sus licitaciones en la Plataforma de Contratación del Sector Público (PCSP) o en los portales o plataformas de contratación propios (España, 2011)

\section{Análisis de los items por territorios autonómicos}

\subsection{Acceso desde la PCSP al perfil de contratante autonómico y su uso como plataforma de publicidad activa}

Los territorios autonómicos pueden publicar sus licitaciones en la PCSP o en los portales o plataformas de contratación propios (España, 2011). Tanto en una como en otra modalidad se cumple el acceso a su perfil de contratante desde la PCSP, $100 \%$ (Gobierno de España, 2018).

La publicidad activa contractual autonómica ha seguido, no obstante, la doble vía señalada: creación de un portal/plataforma de contratación propio o uso de la PCSP.

Casi la mitad de los territorios autonómicos, 9, publican en la PCSP: Aragón, Cantabria, Castilla y León, Castilla-La Mancha, Extremadura, Islas Baleares, Islas Canarias, Melilla y Comunidad Valenciana, el $47,4 \%$.

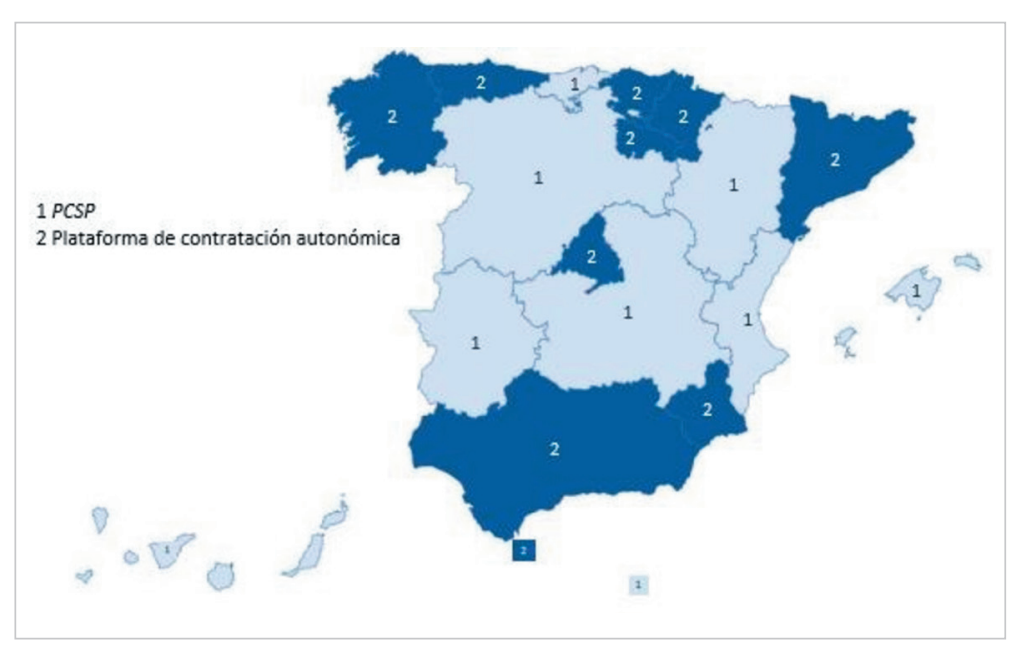

Figura 1. PCSP o plataforma/portal de contratación autonómica. Mapa elaborado con datos de la web de la PCSP 
Poco más de la mitad, 10, han creado su propia plataforma de contratación pública: Andalucía, Asturias, Cataluña, Ceuta, Galicia, La Rioja, Madrid, Murcia, Navarra y País Vasco, el 52,6\% (Gobierno de España, 2018).

Mención especial merece el caso de Islas Baleares. Aunque creó plataforma autonómica, sólo tiene publicadas las contrataciones desde junio de 2008 hasta el 23 de julio de 2017, desde el 24 de julio de 2017 está publicando en la PCSP (Govern Illes Balears, 2018).

\subsection{Acceso al perfil de contratante desde los sitios webs oficiales}

Tal como recoge la tabla 1, 5 territorios autonómicos enlazan el perfil de contratante desde su página de inicio, 10 lo sitúan en un segundo nivel de profundidad al que se accede desde su página de inicio, y uno, Madrid, en un tercer nivel de profundidad. Cumplen $16,84,2 \%$.

Tabla 1. Acceso desde la página de inicio del sitio web oficial, o hasta 3 niveles de profundidad, al perfil de contratante

\begin{tabular}{|c|c|c|}
\hline \multicolumn{2}{|c|}{ Sitios webs oficiales de ciudades y CCAA } & \multirow{2}{*}{$\begin{array}{c}\begin{array}{c}\text { Acceso a su perfil de contratante en página de inicio/otra } \\
\text { página desde un enlace de la página de inicio (segundo nivel } \\
\text { o tercer nivel) }\end{array} \\
\text { No/Sí (segundo nivel) }\end{array}$} \\
\hline Andalucía & https://www.juntadeandalucia.es/index.html & \\
\hline Aragón & http://www.aragon.es & No/Sí (segundo nivel) \\
\hline Asturias & https://www.asturias.es & Sí \\
\hline Cantabria & https://www.cantabria.es & Sí \\
\hline Castilla y León & https://www.jcyl.es & No/Sí (segundo nivel) \\
\hline Castilla-La Mancha & http://www.castillalamancha.es & No/Sí (segundo nivel) \\
\hline Cataluña & http://web.gencat.cat/es/inici/index.html & No/Sí (segundo nivel) \\
\hline Ceuta & http://www.ceuta.es/ceuta/ & No/Sí (segundo nivel) \\
\hline Comunidad Valenciana & http://www.gva.es & Sí \\
\hline Extremadura & https://www.juntaex.es/web & No/Sí (segundo nivel) \\
\hline Galicia & https://www.xunta.gal/portada & No/Sí (segundo nivel) \\
\hline Islas Baleares & http://www.caib.es/govern & No/No \\
\hline Islas Canarias & http://www.gobiernodecanarias.org/principal & No/Sí (segundo nivel) \\
\hline La Rioja & https://www.larioja.org/es & No/No \\
\hline Madrid & http://www.comunidad.madrid/ & No/Sí (tercer nivel) \\
\hline Melilla & http://www.melilla.es & Sí \\
\hline Navarra & https://www.navarra.es & No/No \\
\hline País Vasco & http://www.euskadi.eus & Sí \\
\hline Región de Murcia & http://www.carm.es & No/Sí (segundo nivel) \\
\hline \multicolumn{2}{|l|}{ Total sí / no } & $84,2 \% / 15,8 \%$ \\
\hline
\end{tabular}

Hay 3 autonomías, Islas Baleares, La Rioja y Navarra, que no cumplen este ítem, 15,8\%. En los 3 casos, desde sus sitios web oficiales llevan al apartado "Contratación pública" del portal o plataforma que han adoptado, estatal o autonómica, sin hacer mención expresa al "Perfil de contratante".

\subsection{El Tacrc y sus homólogos autonó- micos}

Para el control del derecho de acceso a la información contractual, publicidad pasiva, existen dos fórmulas: firma de convenio con el Tacrc o creación de un órgano autonómico, tribunal administrativo (España, 2010).

Son 10 los territorios que han firmado convenio con el Tacrc: Asturias, Cantabria, Castilla-La Mancha, Ceuta, Galicia, Islas Baleares, La Rioja, Melilla, Murcia y Comunidad Valenciana, 52,6\%.

Han creado tribunal autonómico 9: Andalucía, Aragón, Castilla y León, Cataluña, Extremadura, Islas Canarias, Madrid, Navarra

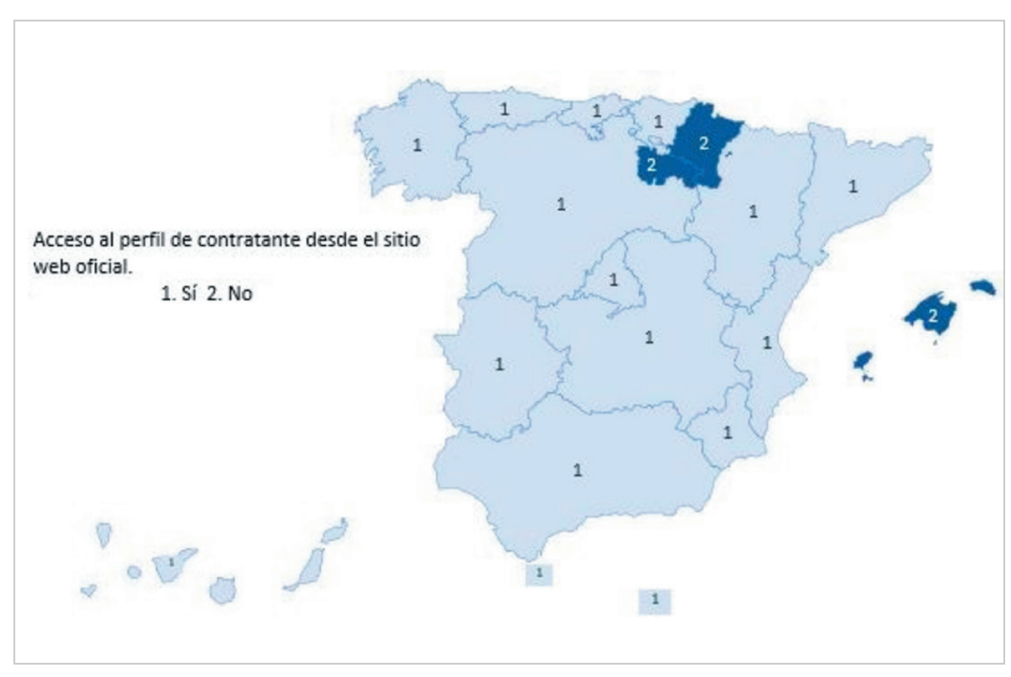

Figura 2. Acceso al perfil contratante desde los sitios web oficiales. Mapa elaborado con los datos de la tabla 1 
y País Vasco, 47,4\% (Ministerio de Hacien$d a, 2018)$.

Aunque coinciden los porcentajes globales con los del apartado 4.2, el porcentaje mayor corresponde en este caso a los que han firmado acuerdo, y tampoco son los mismos territorios los que han optado por la autogestión.

\subsection{El CTBG y sus homólogos autonó- micos}

El control de la publicidad activa ha seguido, también, dos recorridos distintos: firma de convenio con el CTBG o creación del organismo independiente autonómico (España, 2013).

Han firmado convenio 8 de los territorios autonómicos: Asturias, Cantabria, CastiIla-La Mancha, Ceuta, Extremadura, La Rioja, Madrid y Melilla, 42\%.

Mientras, 11 han creado organismo: Andalucía, Aragón, Asturias, Castilla y León, Cataluña, Galicia, Islas Baleares, Islas Canarias, Murcia, Navarra, País Vasco y Comunidad Valenciana, 58\% (CTBG, 2018).

Sube, así, el porcentaje de autonomías que ejercen el control de su publicidad activa con respecto a la pasiva contractual, pero vuelven a no coincidir en todos los casos con los del apartado 4.1 (PCSP), y tampoco lo hacen con los del 4.3 (TacrC).

\subsection{Acceso al perfil de contratante desde el PT o los portales de transpa- rencia autonómicos}

Todos los territorios autonómicos dan acceso a sus perfiles contratantes desde los apartados de contratación de sus portales de transparencia, salvo Islas Baleares, La Rioja y Navarra, 15,8\%, que, como se indicó en el apartado 4.2, omiten el término "Perfil de contratante". El porcentaje de cumplimiento es del 84,2\%. El mapa resultante es el mismo que en el apartado 4.2.

\section{Resultados. Cumplimiento y relación con el Estado ${ }^{3}$}

Los resultados se han reunido en dos grupos temáticos.

El primero es el que se refiere en exclusiva a la publicidad del perfil de contratante:

- desde el sitio web oficial autonómico (apartado 4.2);

- desde el portal de transparencia autonómico (apartado 4.5).

El segundo grupo es el relativo al progreso autonómico:

- las plataformas de publicidad activa contractual (apartado 4.1);

- organismos de control de la publicidad activa contractual (apartado 4.4.) y órganos de control de publicidad pasiva contractual (apartado 4.3)

\subsection{Cumplimiento de publicidad del perfil de contratante}

Los dos items del perfil de contratante miden si se cumple el acceso al perfil de contratante desde el sitio web oficial (España, 2007; 2011) y desde el portal de transparencia autonómico (España, 2013). La importancia de este cumplimiento reside, como se dijo en el apartado 2 , en su reforzado papel en publicidad activa en la última ley de contratos del sector público:

"Los órganos de contratación difundirán exclusivamente a través de internet su perfil de contratante, como ele- 
mento que agrupa la información y documentos relativos a su actividad contractual al objeto de asegurar la transparencia y el acceso público a los mismos" (España, 2017, artículo 63).

Islas Baleares, La Rioja y Navarra no recogen el perfil de contratante en ninguna página de sus sitios web oficiales. En ellos aparece "Contratación pública", que conecta directamente con las plataformas de contratación, estatal o autonómicas. En el caso de La Rioja y Navarra, autonómicas, sin que aparezca el perfil de contratante en ninguna. En Islas Baleares, estatal, aparece el perfil de contratante en el sitio web de la PCSP, pero no en su sitio web oficial. Idéntica situación se produce en sus portales de transparencia. El mapa es el mismo que el del apartado 4.2.

Tabla 2. Acceso al perfil de contratante desde los sitios web oficiales y los portales de transparencia autonómicos

\begin{tabular}{|c|c|c|c|}
\hline $\begin{array}{l}\text { Territorios autonó- } \\
\text { micos }\end{array}$ & $\begin{array}{l}\text { Acceso al perfil de contratante } \\
\text { desde su sede web oficial } \\
\text { (apartado 4.2) }\end{array}$ & $\begin{array}{l}\text { Acceso al perfil de contratante } \\
\text { desde el portal de transparencia } \\
\text { autonómico (apartado 4.5) }\end{array}$ & Cumplimiento autonómico \% \\
\hline Andalucía & $x$ & $x$ & 100 \\
\hline Aragón & $x$ & $x$ & 100 \\
\hline Asturias & $x$ & $\mathrm{X}$ & 100 \\
\hline Cantabria & $x$ & $x$ & 100 \\
\hline Castilla y León & $x$ & $x$ & 100 \\
\hline Castilla-La Mancha & $x$ & $x$ & 100 \\
\hline Cataluña & $x$ & $x$ & 100 \\
\hline Ceuta & $x$ & $x$ & \\
\hline Comunidad Valenciana & $x$ & $x$ & 100 \\
\hline Extremadura & $x$ & $x$ & 100 \\
\hline Galicia & $x$ & $x$ & 100 \\
\hline Islas Baleares & & & 0 \\
\hline Islas Canarias & $x$ & $x$ & 100 \\
\hline La Rioja & & & 0 \\
\hline Madrid & $x$ & $x$ & 100 \\
\hline Melilla & $x$ & $x$ & 100 \\
\hline Navarra & & & 0 \\
\hline País Vasco & $x$ & $\mathrm{x}$ & 100 \\
\hline Región de Murcia & $X$ & $x$ & 100 \\
\hline Total \% & $84,2 \%$ & $84,2 \%$ & \\
\hline
\end{tabular}

Así, Islas Baleares, La Rioja y Navarra no están dando traslado en sus sitios web oficiales ni en sus portales de transparencia del acceso al "Perfil de contratante" recogido en las normas correspondientes (España, 2007; 2011; 2013; 2017).

\subsection{Relación con el Estado}

La relación que han establecido los territorios en materia de contratación con el Estado se ha medido en los items:

- creación de una plataforma de contratación autonómica o el uso de la PCSP, para la publicidad activa, apartado 4.1 (España, 2011);

- creación de un órgano para el control de la publicidad pasiva contractual, tribunal administrativo autonómico, o la firma de un convenio con el Tacrc, apartado 4.3 (España, 2010; 2017);

- creación de un organismo independiente autonómico para el control de la publicidad activa contractual, o la firma de un convenio con el CTBG, apartado 4.4 (España, 2013).

Aunque el total de la casilla del porcentaje de progreso autonómico sea el mismo en muchos casos, no se corresponde siempre con las mismas iniciativas, como veremos a continuación. Ese hecho ha propiciado la reunión de los territorios en 8 modelos:

1. Andalucía, Cataluña, Navarra y País Vasco han asumido tanto la publicidad activa como el control sobre la activa y la pasiva contractuales. Han creando su propia plataforma de contratación, su organismo autónomo de control de la publicidad activa y su órgano de control de la pasiva, obteniendo un progreso autonómico contractual del $100 \%$.

2. Aragón, Castilla y León e Islas Canarias controlan las publicidades activa y pasiva. Publican en la PCSP. Su progreso autonómico en contratación es del $66,6 \%$.

La relación que han establecido los territorios autonómicos en materia de contratación, según tengan plataformas y tribunales propios o firmen convenios con el Estado, ha dado lugar a ocho modelos 
Tabla 3. Plataformas, organismos y órganos de control autonómicos de publicidad contractual

\begin{tabular}{|c|c|c|c|c|}
\hline Territorios autonómicos & $\begin{array}{l}\text { Portal/Plataforma de } \\
\text { contratación autonómi- } \\
\text { co (apartado 4.1) }\end{array}$ & $\begin{array}{l}\text { Tribunal administrativo } \\
\text { de contratación autonó- } \\
\text { mico (apartado } 4.3 \text { ) }\end{array}$ & $\begin{array}{l}\text { Organismo autonómi- } \\
\text { co independiente de } \\
\text { control de publicidad } \\
\text { general (apartado 4.4.) }\end{array}$ & $\begin{array}{c}\text { Progreso autonómico en } \\
\text { materia de contratación } \\
\text { pública } \%\end{array}$ \\
\hline Andalucía & $x$ & $x$ & $x$ & 100 \\
\hline Aragón & & $x$ & $x$ & 66,6 \\
\hline Asturias & $x$ & & & 33,3 \\
\hline Cantabria & & & & 0 \\
\hline Castilla y León & & $x$ & $x$ & 66.6 \\
\hline Castilla-La Mancha & & & & 0 \\
\hline Cataluña & $x$ & $x$ & $x$ & 100 \\
\hline Ceuta & $x$ & & & 33,3 \\
\hline Comunidad Valenciana & & & $x$ & 33,3 \\
\hline Extremadura & & $x$ & & 33,3 \\
\hline Galicia & $x$ & & $x$ & 66,6 \\
\hline Islas Baleares & & & $x$ & 33,3 \\
\hline Islas Canarias & & $x$ & $x$ & 66,6 \\
\hline La Rioja & $x$ & & & 33,3 \\
\hline Madrid & $x$ & $x$ & & 66,6 \\
\hline Melilla & & & & 0 \\
\hline Navarra & $x$ & $x$ & $x$ & 100 \\
\hline País Vasco & $x$ & $x$ & $x$ & 100 \\
\hline Región de Murcia & $x$ & & $x$ & 66,6 \\
\hline Total \% & $52,6 \%$ & $47,0 \%$ & $58,0 \%$ & \\
\hline
\end{tabular}

3. Galicia y Murcia se han quedado con la publicidad activa en contratación y su organismo de control, y firmado convenio con el Tacrc, lo que representa un progreso autonómico contractual del 66,6\%.

4. Madrid ha creado la plataforma de publicidad activa contractual y ha asumido el control de la pasiva, y firmado convenio con el CTBG. Su progreso autonómico contractual es del $66,6 \%$.

5. Asturias, Ceuta y La Rioja han asumido la publicidad activa contractual y firmado convenios con el CTBG y el Tacrc. Su progreso autonómico contractual se sitúa en el 33,3\%.

Andalucia, Cataluña, Navarra y País Vasco. Han creado la plataforma y el organismo de control de publicidad activa, y el órgano de control de publicidad pasiva. $100 \%$

Aragón, Castilla y León e Islas Canarias. Han creado el organismo d control de publicidad activa y el órgano de control de publicidad pasiva. Publican en la PCSP. $66.6 \%$

Galicia y Región de Murcia. Han creado la plataforma y el organismo de control de publicidad activa. Convenio con el Tacrc. $\mathbf{6 6 . 6 \%}$

Madrid. Ha creado la plataforma de publicidad activa y el órgano de control de publicidad pasiva. Convenio con el CTBG. $66.6 \%$

Asturias, Ceuta y La Rioja. Sólo han creado la plataforma de publicidad activa. Tienen convenio con el CTBG y el Tocrc 33.3\%

Extremadura sólo ha creado el órgano de control de publicidad pasiva. Publica en la PCSP y tiene convenio con el CTBG. $33.3 \%$

Comunidad Valenciana e Islas Baleares. Sólo han creado el organismo de control de publicidad activa. Publican en la PCSP y tienen convenio con el Tacrc. 33.3\%

Cantabria, Castilla-La Mancha y Melilla. Publican en la PCSP y tienen convenio con el CTBG y el Tacrc. $0 \%$

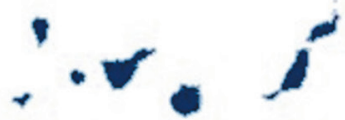

Figura 5. Mapa del progreso autonómico. Plataformas y organismos de control de la publicidad activa y órganos de control de la publicidad pasiva de contratación pública autonómicos.

Mapa elaborado con la información de la tabla 3 
6. Valencia e Islas Baleares han creado el organismo de control de publicidad activa, publican en la PCSP y han firmado convenio con el Tacrc. Su progreso autonómico contractual es del 33,3\%.

7. Extremadura sólo ha creado el tribunal administrativo, publica en la PCSP y tiene convenio con el CTBG. Progreso autonómico contractual del 33,3\%.

8. Cantabria, Castilla-La Mancha y Melilla publican en la PCSP y tienen convenios con el CTBG y el Tacrc. No tienen progreso autonómico contractual.

\section{Conclusiones y propuestas de mejora}

Los progresos autonómicos en materia de publicidad activa y control de publicidades activa y pasiva contractuales han configurado un mapa con 8 modelos presentes en el Estado español (gráfico 5). Este mapa responde a las relaciones establecidas con el Estado más que a las posibles diferencias en la concreción autonómica de la transparencia, como queda reflejado en los casos de Ceuta, Madrid y País Vasco. Ninguna dispone de norma autonómica de transparencia (Gobierno de España, 2018) y, sin embargo, Madrid y País Vasco han hecho progresos autonómicos en materia de contratación del $66,6 \%$ y el $100 \%$, respectivamente.

Las razones de esta diversidad habría que buscarlas, a nuestro juicio, desde otras perspectivas como la idiosincrasia de cada territorio autonómico desde su creación actual, gobiernos y circunstancias concretas por las que ha ido pasando, así como sus relaciones particulares con el Estado. Enfoques como el de las autonomías históricas se deshacen ante realidades como la de Galicia, que no se reúne con las restantes así denominadas en el modelo que ha alcanzado el $100 \%$ en el progreso autonómico en materia de contratación (publicidad activa y control de las publicidades activa y pasiva). Tampoco son válidas las interpretaciones geográficas de proximidad, pues hay 4 modelos con territorios distantes entre sí:

- Aragón, Castilla y León e Islas Canarias: 66,6\%, control de las publicidades activa y pasiva;

- Galicia y Región de Murcia: 66\%, publicidad activa y su control;

- Asturias, Ceuta y La Rioja: 33,3\%, publicidad activa;

- Cantabria, Castilla-La Mancha y Melilla: 0\%.

O los casos de Madrid (66,6\%, publicidad activa y control de la pasiva) y Extremadura (33,3\%, control de la publicidad pasiva), cuyos progresos son únicos ambos. Y el de Islas Baleares y Comunidad Valenciana (33,3\%, control de la publicidad activa), que se unen en un modelo compartido y exclusivo para ambas, con la reciente renuncia de la primera a su publicidad activa contractual.

Por otra parte, a los dos modelos resultantes de este estudio en materia de control de la publicidad activa, CTBG u organismo autonómico, hay que sumarle la disparidad de modelos de atribuciones y configuraciones de los distintos organismos de control autonómico (Sierra-Rodríguez, 2017). En cuanto a la pasiva, tras la entrada en vigor de la Ley 9/2017, de 8 de noviembre, de contratos

Con la creación de los tribunales de ayuntamientos y diputaciones provinciales, es de esperar que el problema se agravará al aumentar la disparidad de criterios, dentro incluso de cada territorio autonómico del sector público (España, 2017), el derecho de acceso a la información deja de estar sujeto a la dualidad CTBG/organismos autonómicos, puesto que este organismo es el único que puede emitir informes que eximan de publicar algunos datos a las administraciones públicas. No obstante, queda igualmente sujeto a la dualidad Tacrc/tribunales autonómicos, y los distintos criterios de los autonómicos más el estatal, ya que no existe una estructura piramidal que unifique criterios en todo el Estado. Con la creación de los tribunales de ayuntamientos y diputaciones provinciales, es de esperar que el problema se agravará al aumentar la disparidad de criterios, dentro incluso de cada territorio autonómico.

La multiplicación de organismos de control y la diversidad de modelos autonómicos ofrecen un mapa de transparencia que, a priori, no parece garantizar el mayor cumplimiento del derecho de acceso a la información contractual, puesto que dificulta su seguimiento global "para analizar y controlar los procesos sustantivos de contratación" (Ochsenius-Robinson, 2017, p. 121). Llegando a darse casos como los de Islas Baleares, La Rioja y Navarra, que obvian el papel clave del perfil de contratante en la publicidad activa según aparece en la normativa. Por otra parte, este complejo escenario puede llevar a situaciones como, por ejemplo, que pequeñas empresas con posibilidades reales de concursar en varias autonomías, tanto en concursos autonómicos como estatales en esos territorios, o incluso locales, tengan que quintuplicar esfuerzos al encontrarse con distintos criterios por territorio y a nivel estatal. Es de esperar que esta realidad se complique más cuando se creen los tribunales administrativos de grandes ayuntamientos y diputaciones provinciales, pues se multiplicarán los criterios en cada territorio. Todo lo anterior apunta, además, a que sólo las empresas de gran tamaño podrán hacer frente a la diversidad, perdiéndose el principio de igualdad de oportunidades perseguido.

Con la creación de los tribunales de ayuntamientos y diputaciones provinciales, es de esperar que el problema se agravará al aumentar la disparidad de criterios, dentro incluso de cada territorio autonómico 
Como propuesta de mejora, y en aras de que los principios de igualdad de oportunidades que promete la transparencia se cumplan, se deberían adoptar algunas medidas:

En primer lugar, dar a las resoluciones de los organismos de control de la publicidad activa, CTBG y autonómicos, así como a los órganos de control de la pasiva, Tacrc y autonómicos, y los de ámbito local que se creen, el carácter de soft law de las Declaraciones que 'proclaman estándares que, sin ser vinculantes, imponen obligaciones morales' (Unesco, 2017) $)^{4}$ y no el potestativo que tiene el Tacrc en la actualidad (Pardo-García-Valdecasas, 2010, p. 23).

Se propone una posible vía de solución futura, la armonización de criterios mediante el concepto de soft law de la Unesco. El soft law, aun no existiendo "unanimidad al respecto de su conceptualización y de las funciones que debe desempeñar" (Alarcón-García, 2010, p. 295) y, como afirma Sarmiento, "cuyas normas no siempre gozan de efectos jurídicos nítidos" (2006, p. 224), sí que concita

"cierto consenso en relación con su catalogación como un fenómeno que comprende aquellos instrumentos que carecen de efectos vinculantes pero que, sin duda, producen efectos jurídicos" (Alarcón-García, 2010, p. 295).

Entendiendo el soft law como instrumentos que "cuando los actores emiten actos o acuerdos no vinculantes lo hacen con el ánimo de cumplirlos y observarlos en un futuro" (Alarcón-García, 2010, p. 296), se podría articular un sistema de cumplimiento y seguimiento global de la transparencia en todo el Estado, al facilitar directrices concretas que impongan obligaciones morales para todos los territorios (vía convenios territoriales, por ejemplo), como medida de optimización o maximización de resultados (Sarmiento, 2006, p. 225).

El carácter de soft law de las resoluciones de los organismos de autocontrol de la publicidad activa haría disminuir, previsiblemente, el número de recursos de la pasiva y proporcionaría directrices claras de publicidad en los procesos de contratación, y seguramente en el resto de la publicidad. Sería una forma de autorregulación de la propia Administración Pública en aras de la transparencia.

De forma paralela, y también para garantizar el cumplimiento de los principios fundamentales de la transparencia en todo el Estado español, se presenta como imprescindible la armonización de los criterios empleados por los organismos de control de la publicidad activa y los órganos de control de la pasiva. Dicha armonización podría plantearse de forma similar a la judicial, siendo en este caso el CTBG y el Tacrc las últimas instancias de reclamación y recurso en publicidades activa y pasiva contractuales, respectivamente. Esta armonización de criterios, vía el carácter de soft law de sus resoluciones, permitiría hacer un seguimiento global de la transparencia en todo el Estado, por una parte, y, por otra, facilitaría su conocimiento y su cumplimiento al haber directrices concretas que impongan obligaciones morales para todos los territorios.

\section{Notas}

1. Se entiende por publicidad activa aquella información que las entidades públicas, o privadas, deben hacer pública en sus sitios web ad hoc, según recoge la Ley 19/2013, de 9 de diciembre, de transparencia, acceso a la información pública y buen gobierno. Es responsabilidad de esas entidades hacerla pública, y corresponde al Consejo de Transparencia y Buen Gobierno, o sus homólogos autonómicos, atender las reclamaciones que se produzcan, e instar a las entidades correspondientes a hacerla pública, salvo en el caso de la información contractual que corresponde al Tribunal Administrativo Central de Recursos Contractuales, o sus homólogos autonómicos o locales.

La publicidad pasiva, derecho a la información, es aquella información no pública que los ciudadanos pueden solicitar a esas mismas entidades, al amparo de dicha ley, exceptuando los casos recogidos en la misma (por seguridad, interés, etc.). Corresponde atender las solicitudes a esas mismas entidades, y sus reclamaciones al Consejo de Transparencia y Buen Gobierno, o sus homólogos autonómicos, salvo en el caso de la información contractual que corresponde al Tribunal Administrativo Central de Recursos Contractuales, o sus homólogos autonómicos o locales.

2. Aunque dos de las disposiciones se encuentran actualmente derogadas: Dar acceso a su perfil de contratante desde la PCSP y publicar sus licitaciones en ella o en las autonómico (España, 2007), y Tener un órgano para el control del derecho de acceso a la información contractual, publicidad pasiva, el Tacrc o el autonómico (España, 2010), los items analizados se han visto reforzados, en la misma línea seguida en este análisis, por normas posteriores y en vigencia (España, 2010) y (España, 2017), respectivamente.

3. Una parte de los resultados de este estudio fueron presentados en el III Congreso internacional de transparencia, celebrado del 26 al 28 de septiembre de 2018 en la Facultad de Ciencias Económicas y Empresariales de la Universidad de Cádiz.

4. El artículo 19 de la Declaración Universal de Derechos Humanos dice: 
“Todo individuo tiene derecho a la libertad de opinión y de expresión; este derecho incluye el de no ser molestado a causa de sus opiniones, el de investigar y recibir informaciones y opiniones, y el de difundirlas, sin limitación de fronteras, por cualquier medio de expresión".

http://www.un.org/es/universal-declaration-human-rights

\section{Referencias}

Alarcón-García, Gloria (2010). "El soft law y nuestro sistema de fuentes”. En: Arrieta-Martínez-de-Pisón, Juan; Collado-Yurrita, Miguel-Ángel; Zornoza-Pérez, Juan (dirs.); Báez-Moreno, Andrés; Jiménez-Valladolid-de-L’Hotellerie-Fallois, Domingo-Jesús (coords.). Tratado sobre la Ley general tributaria: Homenaje a Álvaro Rodríguez Bereijo, v. 1. Pamplona: Ed. Thomson Reuters Aranzadi, pp. 271-298. ISBN: 9788499034836

Beltrán-Orenes, Pilar; Martínez-Pastor, Esther (2016). “Grado de cumplimiento de las Leyes de transparencia, acceso y buen gobierno, y de reutilización de los datos de contratación de la Administración central española". El profesional de la información, v. 25, n. 4, pp. 557-567.

https://doi.org/10.3145/epi.2016.jul.05

Blanes-Climent, Miguel-Ángel (2014). La transparencia informativa de las administraciones públicas. Pamplona: Ed. Thomson Reuters Aranzadi. ISBN: 9788490593974

Canales-Gil, Álvaro; Huertas-Baraja, Justo-Alberto (2010). La contratación del servicio público tras las reformas de 2010. Madrid: Reus. ISBN: 9788429016352

Cerrillo-Martínez, Agustí (2018). “Datos masivos y datos abiertos para una gobernanza inteligente". El profesional de la información, v. 27, n. 5, pp. 1128-1135.

https://doi.org/10.3145/epi.2018.sep.16

Curto-Rodríguez, Ricardo (2017). “Los portales autonómicos de datos abiertos y la información relacionada con la rendición de cuentas: Punto de partida y situación tras la entrada en vigor de la ley 19/2013 de transparencia, acceso a la información pública y buen gobierno". Revista española de la transparencia, n. 5, pp. 80-93.

https://drive.google.com/file/d/1xwjVdVxi5LbNni25Rn-ZJbsKonD-iZsZ/view

Carrascosa-Puertas, Lara (2017). "Transparencia y rendición de cuentas en las páginas webs de los ayuntamientos canarios de más de 20.000 habitantes". Revista española de la transparencia, n. 5, pp. 94-114.

https://drive.google.com/file/d/118HNavw1MedGUScFrEfO1/6HyiM65icF/view

CTBG (2018). Portal de Transparencia. Información económica, presupuestaria y estadística. Convenios de colaboración. Convenios con las Comunidades Autónomas. Consejo de Transparencia y Buen Gobierno.

https://www.consejodetransparencia.es/ct_Home/portal_transparencia/informacion_econ_pres_esta/convenios/ conveniosCCAA.html

España (2007). “Ley 30/2007, de 30 de octubre, de contratos del Sector público”. [Disposición derogada]. BOE, n. 261, 31 octubre, pp. 44336-44436.

https://www.boe.es/buscar/doc.php?id=BOE-A-2007-18874

España (2010). “Ley 34/2010, de 5 de agosto, de modificación de las Leyes 30/2007, de 30 de octubre, de contratos del sector público, 31/2007, de 30 de octubre, sobre procedimientos de contratación en los sectores del agua, la energía, los transportes y los servicios postales y 29/1998, de 13 de julio, reguladora de la Jurisdicción Contencioso-Administrativa para adaptación a la normativa comunitaria de las dos primeras”. BOE, n. 192, 9 agosto, pp. 69400-69433.

https://www.boe.es/buscar/doc.php?id=BOE-A-2010-12765

España (2011). "Real decreto legislativo 3/2011, de 14 de noviembre, por el que se aprueba el texto refundido de la Ley de contratos del sector público". [Disposición derogada]. BOE, n. 276, 16 noviembre, pp. 117729-117914.

https://www.boe.es/buscar/doc.php?id=BOE-A-2011-17887

España (2013). “Ley 19/2013, de 9 de diciembre, de transparencia, acceso a la información pública y buen gobierno". $B O E$, n. 295, 10 diciembre, pp. 97922-97952.

https://www.boe.es/buscar/doc.php?id=BOE-A-2013-12887

España (2017). "Ley 9/2017, de 8 de noviembre, de contratos del sector público, por la que se transponen al ordenamiento jurídico español las Directivas del Parlamento Europeo y del Consejo 2014/23/UE y 2014/24/UE, de 26 de febrero de 2014". BOE, n. 272, 9 noviembre, pp. 107714-108007.

https://www.boe.es/buscar/doc.php?id=BOE-A-2017-12902

Fuentes-Bargues, José-Luis; González-Gaya, Cristina; González-Cruz, María-Carmen (2015). “La contratación pública de obras: situación actual y puntos de mejora”. Informes de la construcción, v. 67, n. 537, e058.

https://doi.org/10.3989/ic.12.130 
García-García, Jesús; Curto-Rodríguez, Ricardo (2018). “Divulgación de información pública de las comunidades autónomas españolas (2013-2017): portal de datos abiertos, portal de transparencia y web institucional". El profesional de la información, v. 27, n. 5, pp. 1051-1060.

https://doi.org/10.3145/epi.2018.sep.09

Gobierno de España (2018). Portal de la transparencia. Comunidades autónomas.

https://transparencia.castillalamancha.es/transparencia/otras-comunidades-autonomas

González-Alonso, Augusto (2009). “La contratación pública”. Cuadernos de derecho público, n. 37, pp. 139-175.

https://revistasonline.inap.es/index.php?journal=CDP\&page=article\&op=view\&path\%5B\%5D=9856\&path\%5B\%5D=10067

González-Varas-Ibáñez, Santiago (2010). "La normativa europea procesal de contratación pública y el sistema actual de recursos contractuales”. Documentación administrativa, n. 288, pp. 43-63.

https://revistasonline.inap.es/index.php?journal=DA\&page=article\&op=view\&path\%5B\%5D=9985\&path\%5B\%5D=10302

Govern Illes Balears (2018). Portal de contratación de las Islas Baleares.

https://www.caib.es/sites/contractaciopublica/es/inicio/?campa=yes

Guichot, Emilio (2014). "La nueva ley de transparencia, un reto para la gestión de las organizaciones públicas". Revista vasca de gestión de personas y organizaciones públicas, n. 6, pp. 94-107.

https://idus.us.es/xmlui/bitstream/handle/11441/59569/La\%20nueva\%20ley....pdf

Martínez-Fernández, José-Manuel (2017). “Nueva Ley de contratos del sector público. Integridad y estrategia como objetivos imperativos". Consultor de los ayuntamientos y de los juzgados: Revista técnica especializada en administración local y justicia municipal, n. 23, (ejemplar dedicado a: Ley de Contratos del Sector Público 2017. Hacia una contratación socialmente eficiente), pp. 2766-2768.

https://cutt.ly/6x6XIw

Martínez-Fernández, José-Manuel (2018). “Contratación estratégica e íntegra. Una experiencia práctica en cifras. Análisis de la contratación del Ayuntamiento de Valladolid". Contratación administrativa práctica. Revista de la contratación administrativa y de los contratistas, n. 157.

http://www.obcp.es/index.php/mod.noticias/mem.detalle/id.1268/relcategoria.118/relmenu.2/ chk.6ca27ed85ba006877918ac7a8586b59b

Mellado-Ruiz, Lorenzo (2018). El principio de transparencia integral en la contratación del sector público. Valencia: Tirant lo Blanch. ISBN: 9788491698036

Ministerio de Hacienda (2018). Tribunal Administrativo Central de Recursos Contractuales. Ministerio de Hacienda. Contratación.

http://www.hacienda.gob.es/es-ES/Areas\%20Tematicas/Contratacion/tacrc/paginas/tribunal\%20administrativo\%20 central\%20de\%20recursos\%20contractuales.aspx

Ochsenius-Robinson, Iván (2017). "La transparencia como control y beneficio de una contratación pública presente". Revista española de la transparencia, n. 4, pp. 110-124.

https://drive.google.com/file/d/OBzZV66dM4HCTdWxkYIRsZXAyWUU/view

Pardo-García-Valdecasas, Juan-José (2010). "El Tribunal Administrativo Central de Recursos Contractuales". Documentación administrativa, n. 288, pp. 19-41.

https://doi.org/10.24965/da.v0i288.9984

Pérez-Delgado, Manuel; Rodríguez-Pérez, Rosario (2017). “Las 11 claves de la Ley 9/2017, 8 de noviembre, de contratos del sector público". Diario la ley, n. 9079.

https://diariolaley.laley.es/home/EX0000127709/20171103/Las-11-claves-de-la-Ley-92017-8-de-noviembre-deContratos-del-Sector-Publico

Sarmiento, Daniel (2006). "La autoridad del Derecho y la naturaleza del soft law”. Cuadernos de derecho público, n. 28, pp. 221-261.

Sierra-Rodríguez, Javier (2017). Modelos de Organismos de Garantía de la Transparencia. Revista española de la transparencia, n. 4, pp. 87-97.

https://drive.google.com/file/d/OBzZV66dM4HCTcnNaaVhpa2IPTkE/view

Unesco (2017). Social and human sciences. Más sobre la naturaleza y el estatus de los instrumentos legales y programas. http://www.unesco.org/new/es/social-and-human-sciences/themes/advancement/networks/larno/legal-instruments/ nature-and-status 


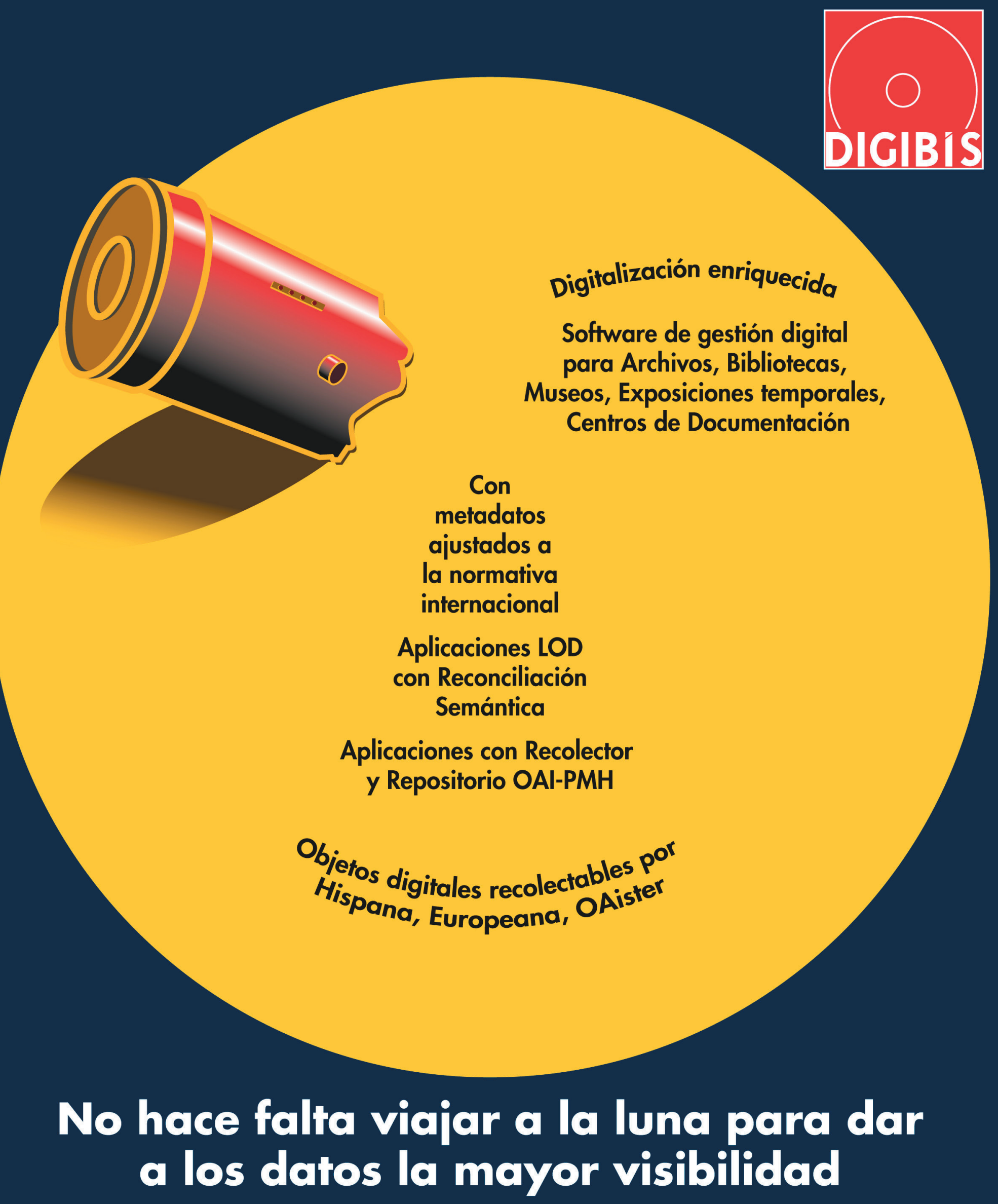

Un concepto de digitalización y unas aplicaciones que hacen más eficiente el trabajo de las instiłuciones de memoria

\section{www.digibis.com}

